such a diagnosis, after adjusting for age, sex, and other chronic conditions. ${ }^{10}$

\section{Managing Care via Prior Authorization (PA) Programs?}

A review of the literature revealed 6 studies in which the prior authorization (PA) method in drug benefit programs was analyzed. ${ }^{11}$ The authors found mixed results on drug costs and little analysis on nondrug medical costs. Equally important, none of the studies examined the effects of PA programs on either service outcomes (satisfaction with care) or health-related quality of life. Only one study, from an inpatient setting, measured other clinical outcomes. The authors concluded that PA programs are commonly used in drug benefit management, but there is (a) no evidence to support their use to manage care and (b) equivocal findings of the effects on drug benefit costs. They also found no PA program benchmarks such as reasonable processing time for a PA, rates of approval of PAs, or measures of administrative costs.

Some HMOs began to drop prior authorization requirements for referrals or medical procedures as early as 1999. Anecdotal and self-reports showed that dropping prior approval (prior authorization) requirements saved administrative cost and reduced member dissatisfaction without having a measurable effect on medical costs. UnitedHealth was among the first to drop prior authorization requirements. In November 1999, UnitedHealth dropped its PA requirements after discovering that 1,200 utilization review nurses denied fewer than $2 \%$ of treatment requests. ${ }^{12}$ Twelve months after the policy change, UnitedHealth found that it had saved $\$ 110$ million in annual administrative costs, had reassigned the UR nurses to other jobs, and experienced a $26 \%$ decline in member complaints to its consumer affairs department. UnitedHealth enjoyed $21 \%$ growth in membership in 2000 and 2001.

In 2001, Blue Cross of California changed its physician and medical group incentive plan from paying bonuses based upon measures of the number of referrals and hospital stays to quality measures that included patient-member satisfaction. UR nurses and other resources assigned to PA programs can be reassigned to quality initiatives such as the program at Harvard Pilgrim in which the nurses monitor and call patients who are at high risk for future illnesses or have not complied with their treatment regimens. The Harvard Pilgrim intervention program recognizes that 1\% of members accounted for $20-25 \%$ of total medical costs.

In this issue of the Journal, Hamer et al. ${ }^{13}$ suggest that gabapentin use be subject to a PA requirement in order to control its use for off-label purposes. This is no idle suggestion. Gabapentin ranked number 16 in community pharmacy sales in CY 2001 at $\$ 1.77$ billion, up 57\% from $\$ 1.13$ billion in CY 2000. ${ }^{14}$ The manufacturer acknowledged that more than $78 \%$ of gabapentin prescriptions in CY 2000 were written for unapproved uses, and there is considerable controversy surrounding the marketing practices and alleged promotion of gabapentin for off-label uses. ${ }^{15,16}$ On May 24, 2002, in the middle of the controversy, the
FDA approved gabapentin for a second indication, management of postherpetic neuralgia in adults. ${ }^{17}$

We need better data on the costs and outcomes of PA programs. At a minimum, we need measures of (a) the rate of approval (number of approvals divided by the number of requests), (b) average processing time per approval, (c) average total administrative cost per denied request, and (d) average total administrative cost per approval. Then, we need measures of clinical outcomes, comparing outcomes among patients denied requests with matched control patients in benefit plans not subject to PA requirements.

\section{Drug Therapy Customized to Individual Patients}

Matching individual genetic information to drug therapy needs may create the opportunity to virtually eliminate adverse drug events (ADEs) and improve the probability of reaching the therapeutic goal in individual patients. It may be possible in as little as 5 years to truly individualize drug therapy. Sounds expensive, and it will be. But, the effective use of genetic information may also permit exclusion of patients from drug therapy that is likely to have little therapeutic effect, or a harmful effect. Applied properly and completely, pharmacogenomics or pharmacogenetics may not be a formidable budget-buster for payers.

Today, clinical drug trials routinely involve collection of patient-specific genetic information. The Mayo Clinic, with business partner International Business Machines, began a project in 2001 to include genomic information in its electronic medical records (EMR) for every patient treated. The project's third phase will record more complex protein data from patients. ${ }^{18}$ Recent work funded by the National Institutes of Health appears to have added another piece to the puzzle related to estrogen replacement therapy (ERT) and its apparent cardioprotective effect in some, but not all, women with coronary artery disease. The research showed that one polymorphism was found in 19\% of the women whose HDL cholesterol levels increased by an average $13 \mathrm{mg}$ per deciliter in response to ERT with conjugated estrogen, an increase more than twice that of patients in the study who did not have the genetic variation. ${ }^{19}$ The researchers concluded that postmenopausal women with coronary disease who have the ER-alpha IVS1-401 C/C genotype, or several other closely related genotypes, have an augmented response of HDL cholesterol to hormone replacement therapy.

This work is not definitive, and the authors acknowledge that there is not yet a reliable link of the responsiveness of HDL cholesterol levels (intermediate outcome) to hormone replacement therapy and the risk or outcome of cardiovascular disease. Nevertheless, this work suggests that pharmacogenetics may provide the possibility to individualize ERT and even allow the common use of genetic tests to help guide most decisions about what drugs and what doses to use in individual patients.

Customized or "personalized" drug treatment is being studied 